\title{
Visible photonic band gap engineering in silicon nitride waveguides
}

\author{
M. C. Nettia) \\ Department of Physics and Astronomy, University of Southampton, SO17 1BJ, United Kingdom \\ M. D. B. Charlton and G. J. Parker \\ Department of Electronics and Computer Science, University of Southampton, Southampton SO17 1BJ, \\ United Kingdom \\ J. J. Baumberg \\ Department of Physics and Astronomy and Department of Electronics and Computer Science, \\ University of Southampton, Southampton SO17 1BJ, United Kingdom
}

(Received 11 October 1999; accepted for publication 21 December 1999)

\begin{abstract}
We demonstrate experimentally the tuning of complete photonic band gaps in patterned silicon nitride waveguides. Transmission measurements were performed using an ultrabroadband high-brightness white light laser continuum, extracting extinction ratios as low as $10^{-4}$ in the gap regions. Angle-resolved measurements show the perfect alignment of the gap around the $\Gamma-J$ direction. (C) 2000 American Institute of Physics. [S0003-6951(00)02008-8]
\end{abstract}

The spatial modulation of the index of refraction in photonic crystals $(\mathrm{PCs})^{1,2}$ has proved to be an effective way to modify the photon density of states. This photonic band gap engineering has numerous and interesting possibilities in optoelectronics. The control of the spontaneous emission, the enhancement of extraction of radiation ${ }^{3,4}$ and the spatial control of the flow of light ${ }^{5}$ are some the main applications of PCs.

Among two-dimensional (2D) PCs, silicon-based waveguides have several attractive features in realizing photonic band gaps (PBGs). First, Si-based technology is already highly exploited in circuit integration. The etching of air rod patterns is a well-controlled and standard technique. Photonic band gaps in these structures arise from the index of refraction contrast between air and Si-based materials and can be tuned by modifying the geometry of the air rods. Second, the waveguide geometry helps to confine light in the high index core region. Finally, although 2D PCs cannot support a full band gap (i.e., a photonic band gap in all directions), the guided modes in the core slab possess gaps which can be significantly engineered. ${ }^{6}$ Recently, 2D GaAsbased PC waveguides have been investigated. ${ }^{7}$ Due to the inclusion of quantum dots actively emitting at fixed wavelength, many samples were needed to explore the photonic band gaps. Moreover, due to the optical pumping geometry, only TE modes could be excited.

In this letter we report an ultrabroadband optical characterization of $2 \mathrm{D}$ silicon nitride $\left(\mathrm{Si}_{3} \mathrm{~N}_{4}\right)$ PCs having a triangular lattice of air rods etched through a waveguide core. Despite their low index contrast, PBGs with high attenuation are obtained in both TE and TM polarization, even though TM gaps are not predicted by simple theories. Simple modification of the structural parameters (pore diameter and lattice pitch) allow the overlap of frequency ranges where both TE and TM guided modes have a band gap, thus creating a complete PBG. The strength of TE and TM PBGs is seen to

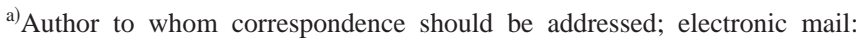
mcnetti@soton.ac.uk
}

be very sensitive to the geometry, since they are formed by different veins and necks in the lattice. ${ }^{8}$

Transmission measurements were performed on two suitably designed sets of PC waveguides. The waveguide layers consisted of a low pressure chemical vapor deposition $\mathrm{Si}_{3} \mathrm{~N}_{4}$ slab $(n=2.02,250 \mathrm{~nm})$ sandwiched between two layers of silicon dioxide $\mathrm{SiO}_{2}$ (75 nm cladding, $1.8 \mu \mathrm{m}$ buffer, $n=1.46) .{ }^{9}$ A triangular lattice of air rods was etched $410 \mathrm{~nm}$ down to the buffer layer in order to create a periodic slab of PC embedded in a symmetric background. ${ }^{6,10}$ The triangular lattice consisted of many rows of air columns with pitches of either $\Lambda=260 \mathrm{~nm}$ or $300 \mathrm{~nm}$ and the same pore radius $r$ $=75 \mathrm{~nm}$ (to avoid changes in etch profile). This choice of the air-filling fraction $f=0.23$ and 0.30 optimized for deep gaps in the near infrared and visible ranges. Smaller holes did not produce appreciable PBGs and larger ones resulted in more extreme scattering of light out of the waveguide as well as being mechanically more fragile.

The laser source used in the measurements was an optical parametric amplifier (Coherent OPA9400) pumped by the output of a regenerative amplifier (Coherent RegA9000) of a Ti:S (Coherent Mira900F). The ultrabroadband white light continuum (WLC) output (450-1100 nm) of the optical parametric amplifier (OPA) was used as well as the signal and idler beams tunable from $440 \mathrm{~nm}$ to $2.3 \mu \mathrm{m}$. In order to improve the achromatic far field collimation, the WLC was focused in a photonic crystal fiber (PCF). ${ }^{11}$ The perforated microstructure of such a fiber results in excellent transmission of the whole WLC spectrum (with a coupling efficiency $\sim 40 \%$ ). The use of the PCF provided excellent beam pointing stability and efficient spectral broadening when the signal and idler pulses were coupled in. The transmittance was obtained by simultaneously recording the input beam spectrum and the transmitted signal with a $0.55 \mathrm{~m}$ monochromator and a charge coupled device (CCD).

The transmission spectra of two representative samples, A ( $\Lambda=300 \mathrm{~nm}, 32$ rows) and B ( $\Lambda=260 \mathrm{~nm}, 40$ rows), are shown in Figs. 1(a) and 1(b), respectively. The transmission is measured in the $\Gamma-J$ direction of the triangular lattice Bril- 

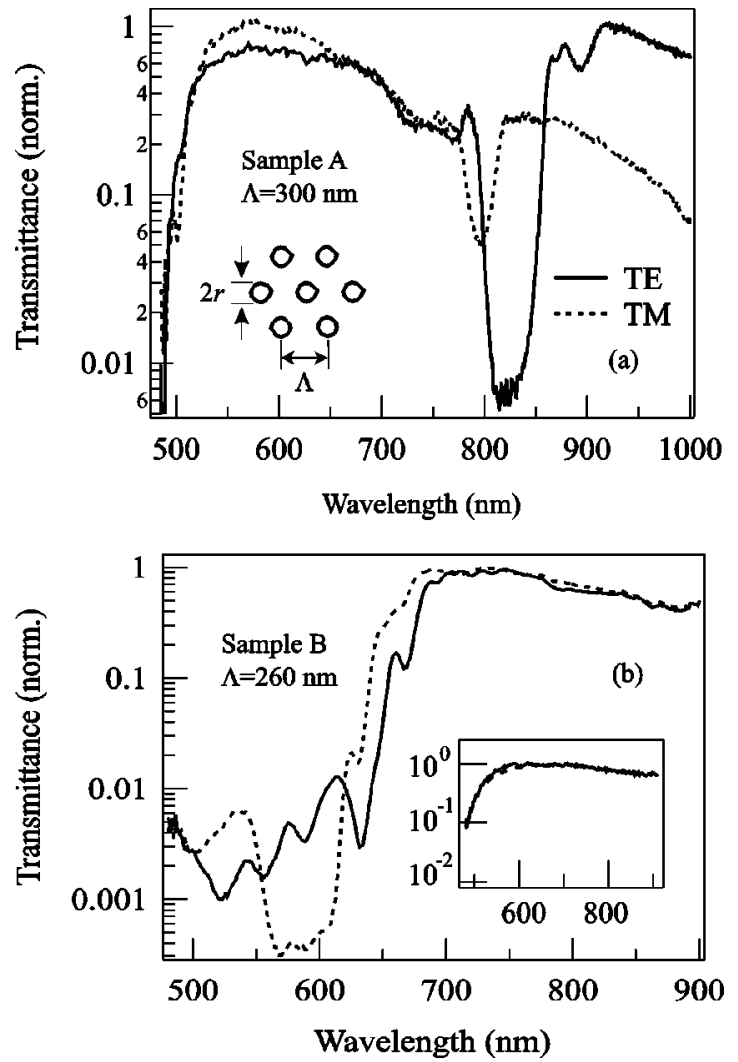

FIG. 1. (a) Transmission spectra of TE (solid line) and TM (dashed line) modes of samples (a) A $(\Lambda=300 \mathrm{~nm}$ pitch and air filling fraction $f$ $=23 \%)$ and (b) B $(\Lambda=260 \mathrm{~nm}$ pitch, $f=30 \%)$. Inset: transmission of a blank $\mathrm{Si}_{3} \mathrm{~N}_{4}$ planar waveguide.

louin zone. Sample A shows sharp gaps in the near infrared for both polarizations which overlap $\sim 50 \%$. The TE gap is centered at $\lambda_{0}=825 \mathrm{~nm}$ and is $90 \mathrm{~nm}$ wide with an extinction ratio of about $6 \times 10^{-3}(42 \mathrm{~dB})$. The normalized width of this gap $\left(\Delta \lambda / \lambda_{0}\right)$ is 0.11 . The TM gap is shallower and shifted to shorter wavelength, centered at $\lambda_{0}=783 \mathrm{~nm}$ with $\Delta \lambda / \lambda_{0}=0.06$ and an extinction ratio of $5 \times 10^{-2}(24 \mathrm{~dB})$. The decrease of transmittance below $550 \mathrm{~nm}$ is due to losses at shorter wavelength evident in the unpatterned planar waveguide (shown in the inset). The broadband WLC also reveals the strong scattering of light both in and out of plane. Wavelength-dependent beam steering occurs in the plane of the core, while a grating-like diffraction occurs out of plane. The out-of-guide scattering patterns are different for TE and TM modes and stronger for the TE mode accounting for the reduced TE transmission. The development of techniques to measure broadband waveguide transmission provides clear data to feed back to both our fabrication strategies and theoretical models.

By decreasing the air column spacing from 300 to 260 $\mathrm{nm}$, several features change in the transmission for both polarizations. The main difference is the shift of the PBG to visible wavelengths as shown in Fig. 1(b). As expected from calculations, an increase of $r / \Lambda$ results in a shift of the PBG towards shorter wavelength. The strong overlap of the band gaps and the greater attenuation of transmission for both TE and TM modes are significant. The PBGs are centered at 585 $\mathrm{nm}$ with a normalized width of 0.33 and 0.19 for TE and TM modes, respectively. As for sample A, the transmission suffers from scattering losses at shorter wavelength. In contrast (a)

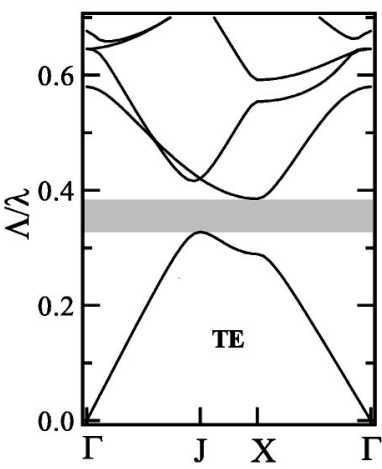

(c)

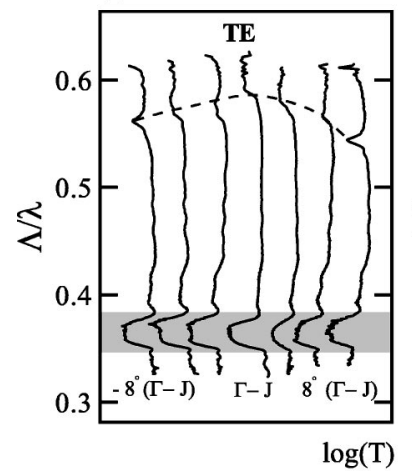

(b)

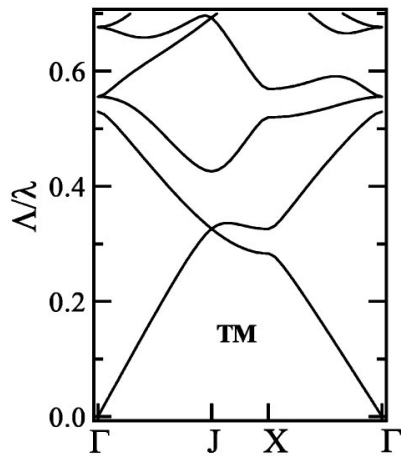

(d)

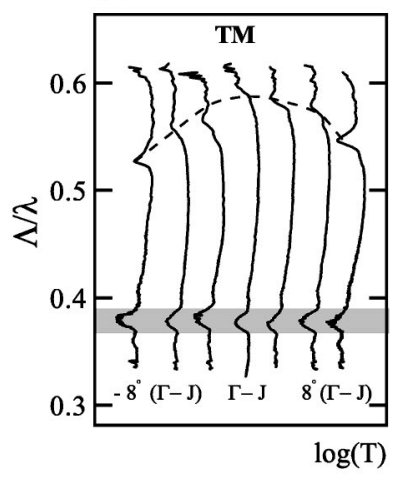

FIG. 2. Two-dimensional dispersion relations for (a) TE and (b) TM polarizations. Angular-resolved transmission for internal angles in the range $\pm 8^{\circ}$ around the $\Gamma-J$ direction for (c) TE and (d) TM, for sample A. In (a), (c), and (d) the shaded area shows the photonic gap. The dashed line is a guide to the eye.

to sample A, the extinction ratio for the TM mode is nearly two order of magnitude larger $\left(3 \times 10^{-4}, 68 \mathrm{~dB}\right)$, and only slightly less for the TE mode $\left(1 \times 10^{-3}, 58 \mathrm{~dB}\right)$. This inversion of attenuation for TE and TM modes produced by reducing the pitch can be attributed to the changing geometry of the high-index necks ${ }^{8}$ that generate TM band gaps. Reducing the pitch reduces the size of the vertices $(23 \mathrm{~nm})$ less than the size of the connecting veins $(40 \mathrm{~nm})$.

The TE band gap of sample B is characterized by the presence of several shallow states (the axis is logarithmic). These are unlikely to be localized states in the gap due to their systematic presence in samples with a very different number of air column rows. ${ }^{12}$ We also checked that they are not due to polarization mixing inside the PC. Shallow states can arise from the dispersion of the guided mode in different symmetry directions. The PC band structures were calculated using both $2 \mathrm{D}$ and three-dimensional (3D) plane wave analysis ${ }^{13}$ showing identical results. ${ }^{9}$ The dispersion relations for 2D calculation are reported in Figs. 2(a) and 2(b) for TE and TM modes. A full band gap is expected only for TE modes in the normalized energy range $\Lambda / \lambda=0.32-0.38$. In Figs. 2(c) and 2(d) the angle resolved transmission for sample A is shown for both TE and TM polarizations, plotted versus $\Lambda / \lambda$ for convenient comparison. The experimental curves were acquired at regular steps in the range $\pm 8^{\circ}$ around the $\Gamma-J$ direction (the angle between the $\Gamma-X$ and $\Gamma-J$ directions is $30^{\circ}$ ). The TE polarization shows a photonic gap in good agreement with the theoretical calculation. As already shown in Fig. 1, the TM polarization also shows 


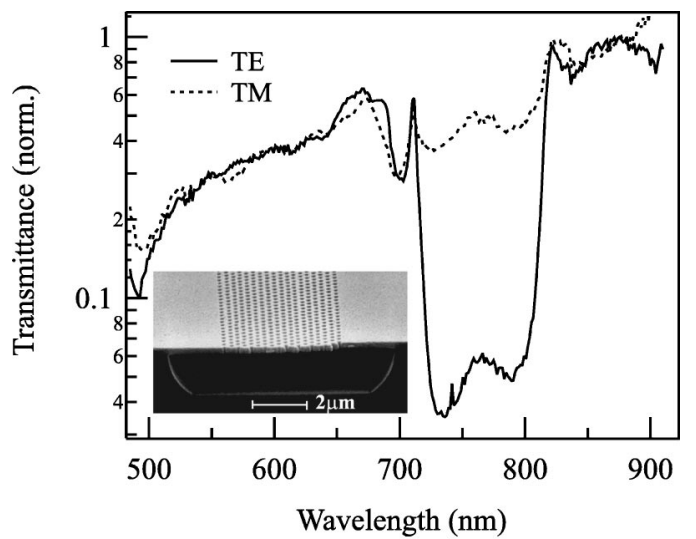

FIG. 3. Transmission spectra of the air bridge structure for TE (solid line) and TM (dashed line) polarizations.

a gap in transmission, confounding the theoretical predictions. For each polarization, the gaps are fixed in the same frequency range and have approximately the same extinction ratio throughout the angles investigated. An unexpected feature is the presence of a dip at $\Lambda / \lambda \sim 0.58$ that shifts its positions towards lower frequencies for propagation away from $\Gamma-J$. The transmission in the dip decreases by nearly an order of magnitude at $\pm 8^{\circ}$. We attribute this dispersion-like behavior to a guided mode. Similar angular tuning was obtained for sample B with PBGs in the normalized frequency ranges $0.40-0.52$ (TE) and 0.42-0.48 (TM). The experimental results in Fig. 2 suggest the inadequacy of a simple 2D or $3 \mathrm{D}$ model neglecting the behavior of the waveguiding slab. As recently reported in Ref. 6 the dispersion of guided modes in a triangular lattice of air holes in a high dielectric medium is strongly dependent on the geometry of the slab and quite different from the modes from a $2 \mathrm{D}$ calculation.

Waveguide models also predict a strong influence from the symmetry of the confinement layers, so transmission was measured on an undercut bridge structure. ${ }^{14}$ The latter was created by etching the underlying buffer creating a $650-\mathrm{nm}-$ thick layer of air underneath the $\mathrm{SiO}_{2}-\mathrm{Si}_{3} \mathrm{~N}_{4}-\mathrm{SiO}_{2}$ structure. The sample was similar to sample A having a pitch $\Lambda$ $=300$ and $r / \Lambda=0.225$. The transmission spectra (Fig. 3) strikingly show the perfect overlap of TE and TM band gaps, in contrast to sample A. They are centered at $765 \mathrm{~nm}$ and shifted towards shorter wavelength. Surprisingly, the extinction ratio is not better than that of sample $\mathrm{A}$, reaching $\sim 5$ $\times 10^{-2}(\mathrm{TE})$ and $5 \times 10^{-1}(\mathrm{TM})$. Another unexpected feature is the presence of a band in the middle of both TE and TM gaps. Its overlap at the same wavelength $(765 \mathrm{~nm})$ might suggest the presence of mode mixing or of a localized state present in the gap.

In conclusion, we have experimentally demonstrated the existence of complete PBGs in $\mathrm{Si}_{3} \mathrm{~N}_{4}$ photonic crystal waveguides using a broadband waveguide characterization. Proper choice of the structural parameters $\Lambda$ and $r$ of the air columns in a triangular lattice allows us to tune the PBGs across the visible and near infrared spectral ranges. The comparison of experimental data with $2 \mathrm{D}$ and $3 \mathrm{D}$ plane-wave analysis shows a satisfactory agreement for TE polarization. A trade-off between the overlap of TE and TM gaps and their relative extinction ratio was found. However, a more complete model of guided modes in PC slabs in progress is necessary to explain the experimental results.

The authors acknowledge the support of the University of Southampton, Coherent UK Ltd., and Hitachi Europe Ltd. This work was partly supported by HEFCE JR98SOBA.

${ }^{1}$ E. Yablonovitch, Phys. Rev. Lett. 58, 2059 (1987).

${ }^{2}$ S. John, Phys. Rev. Lett. 58, 2486 (1987).

${ }^{3}$ S. Fan, R. Villeneuve, J. D. Joannopoulos, and E. F. Schubert, Phys. Rev. Lett. 78, 3294 (1997).

${ }^{4}$ M. Boroditsky, T. F. Krauss, R. Coccioli, R. Vrijen, R. Bhat, and E. Yablonovitch, Appl. Phys. Lett. 75, 1036 (1999).

${ }^{5}$ H. Kosaka, T. Kawashima, A. Tomita, M. Notomi, T. Tanamura, T. Sato, and S. Kawakami, Phys. Rev. B 58, R10096 (1998); H. Kosaka, T. Kawashima, A. Tomita, M. Notomi, T. Tanamura, T. Sato, and S. Kawakami, Appl. Phys. Lett. 74, 1370 (1999).

${ }^{6}$ S. G. Johnson, S. Fan, P. R. Villeneuve, J. D. Joannopoulos, and L. A. Kolodziejski, Phys. Rev. B 60, 5751 (1999).

${ }^{7}$ D. Labilloy, H. Benisty, C. Weisbuch, T. F. Krauss, R. M. De La Rue, V. Bardinal, R. Houdré, U. Oesterle, D. Cassagne, and C. Jouanin, Phys. Rev. Lett. 79, 4147 (1997).

${ }^{8}$ J. D. Joannopoulos, R. D. Meade, and J. N. Winn, Photonic Crystals (Princeton University Press, Princeton, NJ, 1995).

${ }^{9}$ M. D. B. Charlton, S. W. Roberts, and G. J. Parker, Mater. Sci. Eng., B 49, 155 (1997).

${ }^{10}$ J. N. Winn, R. D. Meade, and J. D. Joannopoulos, J. Mod. Opt. 41, 257 (1994); A. Barra, D. Cassagne, and C. Jouanin, Appl. Phys. Lett. 72, 627 (1998).

${ }^{11}$ P. J. Bennett, T. M. Monro, and D. J. Richards, Opt. Lett. 24, 1203 (1999); J. C. Knight, T. A. Birks, P. St. J. Russell, and D. M. Atkin, Opt. Lett. 21, 1547 (1996); J. C. Knight, T. A. Birks, P. St. J. Russell, and D. M. Atkin, Opt. Lett. 21, 1547 (1996).

${ }^{12}$ M. C. Netti, J. J. Baumberg, M. D. B. Charlton, and G. J. Parker (unpublished).

${ }^{13}$ M. Phlihal and A. A. Maradudin, Phys. Rev. B 44, 8565 (1991).

${ }^{14}$ P. R. Villeneuve, S. Fan, J. D. Joannopoulos, K.-Y. L. Lim, G. S. Petrich, L. Kolodziejski, and R. Reif, Appl. Phys. Lett. 67, 167 (1995). 